\title{
CONSUMO DE BENZODIAZEPÍNICOS E OPIOIDES NO PRIMEIRO QUADRIMESTRE DE 2020 EM UMA UNIDADE DE PRONTO ATENDIMENTO DA PARAÍBA
}

CONSUMPTION OF BENZODIAZEPHINES AND OPIOIDS IN THE FIRST FIRST FOUR MONTHS OF 2020 IN A EMERGENCY MEDICAL SERVICES IN PARAÍBA

\author{
Rômulo Moreira dos Santos ${ }^{1}$ \\ Raissa Daniel Trajano dos Santos ${ }^{2}$ \\ Tássya Rebecka Neves Araújo ${ }^{3}$ \\ Luana Layse Câmara de Almeida ${ }^{4}$ \\ Luana Beatriz Camêlo ${ }^{5}$
}

RESUMO: Objetivo: Verificar a dispensação de benzodiazepínicos e analgésicos opioides, classificando-os pelo Anatomic Therapeutic Chemical e quantificando os gastos a partir de valores base da Câmara de Regulação do Mercado de Medicamentos. Método: O estudo foi do tipo transversal, com abordagem quantitativa, desenvolvido na Unidade de Pronto Atendimento de Porte III do município de Campina Grande, durante o mês de abril de 2020. Foram consultadas as planilhas de estoque da Farmácia desse serviço de saúde de urgência e emergência, referentesao primeiro quadrimestre do corrente ano, para verificação do consumo de benzodiazepínicos e opioides dispensados para os pacientes em atendimento na faixa temporal selecionada, sem contato algum, direto ou indireto, com estes pacientes ou suas informações pessoais. Resultados: Foram dispensados um total de 2882 apresentações farmacêuticas dos medicamentos benzodiazepínicos ou opioides, sendo estes últimos motivo de $62,3 \%$ das dispensações. Conclusão: É de grande importância a verificação do uso de medicamentos na sociedade, para melhor entender suas indicações, problemas associados e gastos para manutenção dos tratamentos. Pelo grande número de opioides dispensados em altas concentrações, seria adequada a implementação de

\footnotetext{
1 Farmacêutico, Mestre em Saúde Pública e Professor dos cursos de Farmácia e Nutrição da Unifacisa.

${ }^{2}$ Graduanda do curso de farmácia da Unifacisa.

${ }^{3}$ Graduanda do curso de farmácia da Unifacisa.

${ }^{4}$ Graduanda do curso de farmácia da Unifacisa.

${ }^{5}$ Graduanda do curso de farmácia da Unifacisa.
} 
programas de educação em saúde para os funcionários, que resultassem sempre no melhor atendimento aos usuários do serviço.

Palavras chave: Farmacoepidemiologia. Unidade de Pronto Atendimento. Medicamento de Controle Especial.

ABSTRACT: Objective: To verify the dispensation of benzodiazepines and opioid analgesics, classifying them by Anatomic Therapeutic Chemical and quantifying expenditures based on base values of the Regulation Chamber of the Medicines Market. Method: The study was a cross- sectional study, with a quantitative approach, developed in the Emergency Care Unit of Size III in the municipality of Campina Grande, during the month of April 2020. The Pharmacy stock sheets of this emergency health service were consulted. and emergency, referring to the first four months of this year, to check the consumption of benzodiazepines and opioids dispensed to patients in care in the selected time range, without any direct or indirect contact with these patients or their personal information. Results: $A$ total of 2882 pharmaceutical presentations of benzodiazepine or opioid drugs were dispensed, the latter being the reason for $62.3 \%$ of dispensations. Conclusion: It is of great importance to check the use of medicines in society, to better understand their indications, associated problems and expenses for maintaining treatments. Due to the large number of opioids dispensed in high concentrations, it would be appropriate to implement health education programs for employees, which would always result in better service to service users.

Keywords: Pharmacoepidemiology. Emergency Medical Services. Drug Prescription of Special Control. 


\section{INTRODUÇÃO}

As Unidades de Pronto Atendimento (UPA) são unidades de saúde com funcionamentonas 24 horas dos dias, sendo responsáveis por interligar os serviços de atenção básica, atençãohospitalar, atenção domiciliar e o Serviço de Atendimento Móvel de Urgência - SAMU 192, recebendo os atendimentos de média complexidade, diminuindo a demanda, principalmente, hospitalar (CASSETARI; MELLO, 2017).

Ainda, as UPA devem apresentar um porte mínimo para prestar atendimento essencial aos usuários, sendo exigida estrutura simplificada, com raio-X, eletrocardiografia, pediatria, laboratório de exames e leitos de observação (BRASIL, 2020).

Como nestes serviços são direcionados os atendimentos para febre alta, acima de $39^{\circ} \mathrm{C}$, fraturas e cortes com pouco sangramento, Infarto e derrame, queda com torsão e dor intensa ou suspeita de fratura, cólicas renais, falta de ar intensa, crises convulsivas, dores fortes no peito e vômito constante, fica justificada a disponibilização para prescrição e dispensação de medicamentos que contenham fármacos benzodiazepínicos e analgésicos opioides.

Destes medicamentos, os benzodiazepínicos são prescritos para tratar os quadros de ansiedade e outros distúrbios do humor, além de alguns representantes, como o clonazepam, serem indicados para controlar episódios convulsivos (FEGADOLLI; VARELA, CARLINI, 2019). O Brasil é considerado o país com o maior número de indivíduos diagnosticados com ansiedade, doença responsável por causar sintomas como respiração ofegante e falta de ar, palpitações e dores no peito, tontura, sensação de desmaio, enjoo e vômitos, o que faz muitos pacientes procurarem uma UPA com suspeita de problemas cardiovasculares e lá receberem tratamentos para essa sintomatologia mais aguda (ZWIELEWSKI; BUB, 2014).

No caso dos opioides, são analgésicos de ação central que aliviam a dor atuando sobre células nervosas específicas da medula espinhal e do cérebro, 
estimulando receptores endógenos opioides Mi, Delta e Kapa, responsáveis pelas sensações de analgesia, euforia e sedação (MARTINS et al., 2012).

O conhecimento da utilização de medicamentos que contenham essas substânciasfavorece o entendimento do uso em grandes populações, já que a UPA local de estudo recebe pacientes locais e de municípios circunvizinhos, além de proporcionar dados sobre a farmacoepidemiologia dessas classes e melhorando os achados nos âmbitos da farmacovigilância e farmacoeconomia.

\section{MÉTODO}

O estudo foi do tipo transversal, com abordagem quantitativa, desenvolvido na Unidade de Pronto Atendimento de Porte III do município de Campina Grande, durante o mês de abril de 2020. Foram consultadas as planilhas de estoque da Farmácia desse serviço de saúde de urgência e emergência, referentes ao primeiro quadrimestre do corrente ano, para verificação do consumo de benzodiazepínicos e opioides dispensados para os pacientes em atendimento na faixa temporal selecionada, sem contato algum, direto ou indireto, com estes pacientes ou suas informações pessoais. Um formulário semiestruturado foi utilizado para registro do quantitativo mês a mês, sempre na presença de um colaborador da Farmácia, mantendo a segurança dos registros consultados e garantindo o não vazamento de informações para outros fins não autorizados.

Os medicamentos consultados foram classificados de acordo com 0 Anatomical Therapeutic Chemical (WHO, 2020) e os valores para a aquisição dos produtos, foramcalculados, de maneira aproximada, a partir da consulta a Planilha da Câmara de Regulação doMercado de Medicamentos (CMED) (BRASIL, 2020), que é um órgão ministerial responsável pela regulação econômica destes produtos no Brasil, pertencendo a Secretaria Executiva à Agência Nacional de Vigilância Sanitária.

Os valores consultados na planilha da CMED, são os informados pela taxação, a qual o Estado da Paraíba está submetido, sendo os valores de Preço 
Fábrica (PF) acrescidos da alíquota de $18 \%$ de Imposto sobre Circulação de Mercadorias e Serviços (ICMS).

\section{RESULTADOS E DISCUSSÃO}

Foram dispensados pela Farmácia da UPA consultada, um total de 2882 apresentações farmacêuticas dos medicamentos benzodiazepínicos ou opioides, durante os quatro primeiros meses de 2020. Deste total, o consumo de medicamentos opiáceos representou $62,3 \%$ das dispensações, representando, também, a maior porcentagem dentre todos os medicamentos sujeitos a controle especial. Na Tabela 1, encontram-se as apresentações disponíveis destas classes e suas indicações de uso.

Tabela 1. Apresentações de Benzodiazepínicos e Opioides Disponíveis na UPA Local do Estudo.

\begin{tabular}{|c|c|c|c|}
\hline ATC NÍVEL & $\begin{array}{l}\text { CÓDIGO } \\
\text { ATC }\end{array}$ & APRESENTAÇÃO & $\begin{array}{l}\text { PRINCIPAL } \\
\text { INDICAÇÃO }\end{array}$ \\
\hline \multirow{6}{*}{ Benzodiazepínicos } & N03AE01 & Clonazepam 2mg VO & $\begin{array}{l}\text { Ansiolítico e } \\
\text { anticonvulsivante }\end{array}$ \\
\hline & \multirow{3}{*}{ N05BA01 } & Diazepam 5mg VO & \multirow{3}{*}{ Ansiolítico } \\
\hline & & Diazepam 10mg VO & \\
\hline & & $\begin{array}{l}\text { Diazepam 10mg VO } \\
\text { Diazepam 10mg/mL IM }\end{array}$ & \\
\hline & \multirow{2}{*}{ N05CD08 } & $\begin{array}{l}\text { Midazolam } 15 \mathrm{mg} / 3 \mathrm{~mL} \\
\text { IM/IV }\end{array}$ & \multirow{2}{*}{$\begin{array}{l}\text { Hipnótico } \\
\text { anestésico }\end{array}$} \\
\hline & & $\begin{array}{l}\text { Midazolam } \\
\text { IM/IV }\end{array}$ & \\
\hline \multirow{6}{*}{ Opioides } & \multirow[b]{2}{*}{ N01AH01 } & Fentanila $0,1 \mathrm{mg} / 2 \mathrm{Ml} \mathrm{IM} / \mathrm{IV}$ & \multirow{2}{*}{$\begin{array}{l}\text { Analgésico } \\
\text { anestésico }\end{array}$} \\
\hline & & $\begin{array}{l}\text { Fentanila } \\
\text { IM/IV }\end{array}$ & \\
\hline & \multirow{2}{*}{ N02AA01 } & $\begin{array}{l}\text { Morfina } \\
\text { IM/IV/IT/PD }\end{array}$ & \multirow{2}{*}{$\begin{array}{l}\text { Analgésico para } \\
\text { dor severa }\end{array}$} \\
\hline & & $\begin{array}{ll}\text { Morfina } & 10 \mathrm{mg} / \mathrm{m} \\
\text { IM/IV/IT/PD } & \end{array}$ & \\
\hline & \multirow{2}{*}{ N02AX02 } & Tramadol 50mg/mL IM/IV & \multirow{2}{*}{$\begin{array}{l}\text { Analgésico para } \\
\text { dor moderada a } \\
\text { grave }\end{array}$} \\
\hline & & Tramadol 100mg/2mL IM/IV & \\
\hline
\end{tabular}

Fonte: Dados do estudo, 2020.

Via oral (VO), Intramuscular (IM), Intravenosa (IV), Intratecal (IT), Peridural (PD). 
Dos benzodiazepínicos (BZD), o mais dispensado foi o diazepam, informação que diverge do publicado pela Agência Nacional de Vigilância Sanitária nos relatórios do Sistema Nacional de Gerenciamento de Produtos Controlados, no qual o clonazepam figura como o BZD mais prescrito no Brasil (ANVISA, 2020).

Os quantitativos da dispensação dos medicamentos opioides e BZD estão na Figura 1, estratificados nos quatro primeiros meses deste ano. Nesta figura, observa-se que o tramadol é o opioide mais prescrito, indicando o atendimento de condições dolorosas de intensidade moderada e que não apresentam de um acompanhamento mais prolongado.

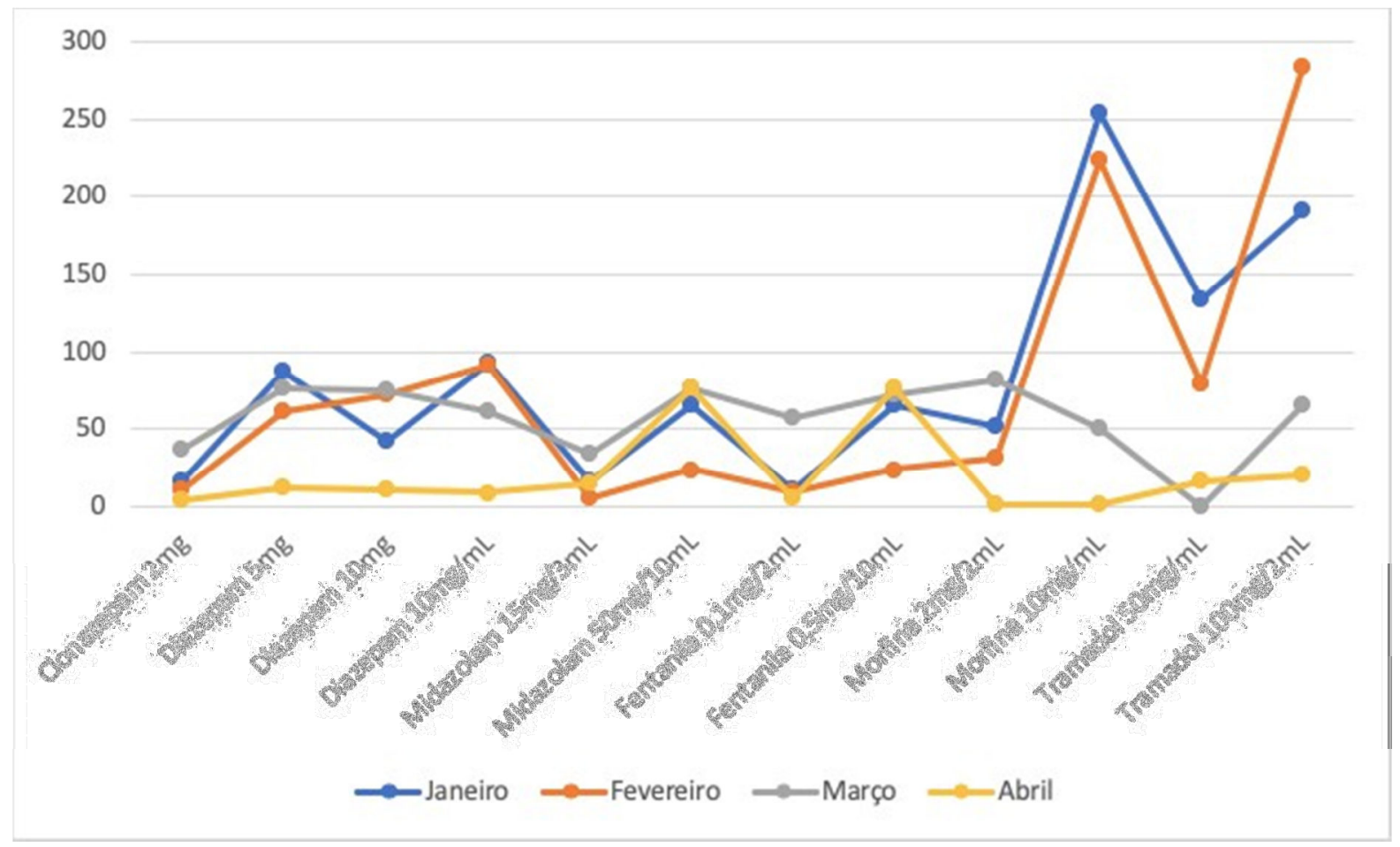

Figura 1. Consumo de Benzodiazepínicos e Opioides no primeiro quadrimestre de 2020 em uma UPA da Paraíba. Dados do estudo, 2020.

No entanto, o consumo de morfina é extremamente elevado na UPA consultada, sendo a apresentação que contém $10 \mathrm{mg}$ do sulfato de morfina a mais dispensada pela farmácia. $O$ grande problema da utilização de analgésicos opioides está nas possíveis reações adversas queestes medicamentos podem causar. 
A depressão respiratória é o evento adverso mais complicado causado por opiáceos, característica que pode agravar sintomas decorrentes de outras doenças e condições apresentadas pelos pacientes com quadros mais graves e internados na ala vermelha da unidade (BICCA et al., 2012).

O consumo geral de medicamentos na UPA deste estudo foi maior nos dois primeiros meses do ano, pois são anteriores ao Decreto Federal que declarou o estado de pandemia devido ao Novo Coronavírus (COVID-19). Após o estado de pandemia, a redução foi considerável, levando aos quantitativos diminuírem mais que $50 \%$.

Este fato também se relaciona a unidade de urgência e emergência consultada ter sido direcionada para recebimento, apenas, de pacientes que apresentassem problemas respiratórios, associados a síndromes gripais.

Neste período, a prescrição de opiáceos foi a mais restringida, pois não configuram como tratamento de escolha para as condições em atendimento (KRAYCHETE et al., 2013), ficando aos demais medicamentos em números mais semelhantes.

Percebe-se que a quantidade do uso de midazolam e fentanila são semelhantes, justificado pela associação proposital para os procedimentos de sedação e entubação de pacientes em estados que requeiram maiores cuidados. Apesar dos possíveis riscos associados a esta interação, a utilização conjunta desses medicamentos faz parte de protocolos clínicos para pacientes em unidades de terapia intensiva, classificados para áreas vermelhas de serviços de saúde (BENSEÑOR; CICARELLI, 2003).

As UPA são divididas em três alas de atendimento, seguindo uma metodologia de triagem por cores: a ala verde para pacientes que podem aguardar atendimento especializado e para a administração de medicamentos em pacientes que recebem alta logo na sequência; a alaamarela para usuários com condições que, apesar da necessidade de atendimento rápido, podem aguardar; e a ala vermelha para os pacientes com necessidade de atendimento imediato. Em quaisquer situações das alas amarela e vermelha, sempre que possível, os pacientes sãoregulados para atendimentos hospitalares. 
Através das planilhas de registros de dispensação, os BZD foram mais dispensados paraa ala verde $(82,7 \%$ - 898) e os opioides em maior quantidade para as alas amarela e vermelha, locais que recebem internações e de pacientes mais graves.

$\mathrm{Na}$ Tabela 2 estão os valores, aproximados, para a compra destes medicamentos no período estudado, baseados na CMED (ANVISA, 2020).

Tabela 2. Despesas Referentes ao Consumo de Benzodiazepínicos e Opioides Padronizados na UPA Local do Estudo, 2020

\begin{tabular}{lccc}
\hline APRESENTAÇÃO & $\begin{array}{c}\text { CONSUMO } \\
\text { MÉDIO } \\
\text { MENSAL }\end{array}$ & $\begin{array}{c}\text { VALOR } \\
\text { UNITÁRIO } \\
\text { R \$ }\end{array}$ & $\begin{array}{c}\text { GASTO } \\
\text { MÉDIO } \\
\text { MENSAL R }\end{array}$ \\
\hline Clonazepam 2mg VO & 17 & 0,56 & 9,52 \\
\hline Diazepam 5mg VO & 60 & 0,27 & 16,20 \\
\hline Diazepam 10mg VO & 50 & 0,37 & 18,50 \\
\hline Diazepam 10mg/mL IM & 64 & 0,56 & 35,84 \\
\hline Midazolam 15mg/3mL IM/IV & 21 & 11,69 & 245,49 \\
\hline Midazolam 50mg/10mL IM/IV & 18 & 31,78 & 572,04 \\
\hline Fentanila 0,1mg/2mL IM/IV & 60 & 1,84 & 110,04 \\
\hline Fentanila 0,5mg/10mL IM/IV & 59 & 5,18 & 305,62 \\
\hline Morfina 2mg/2mL IM/IV/IT/PD & 42 & 5,17 & 217,14 \\
\hline Morfina 10mg/mL IM/IV/IT/PD & 132 & 3,41 & 450,12 \\
\hline Tramadol 50mg/mL IM/IV & 57 & 4,70 & 267,90 \\
\hline Tramadol 100mg/2mL IM/IV & 140 & 6,72 & 940,80 \\
\hline & & & 3189,21 \\
\hline
\end{tabular}

Fonte: Dados do estudo, 2020.

Via oral (VO), Intramuscular (IM), Intravenosa (IV), Intratecal (IT), Peridural (PD).

O gasto total com os medicamentos estudados resultou em Doze mil, setecentos e cinquenta e seis reais e oitenta e quatro centavos ( $R \$ 12.756,84)$, especificamente $\mathrm{R} \$ 3.590,36$ para os $B Z D$ e $R \$ 9.166,48$ para os opiáceos.

Observando as médias de consumo mensal, o tramadol é o medicamento sujeito à controle especial mais prescrito. Apesar de ser classificado como analgésico opioide, sabe-se que sua interação aos receptores opioide do tipo $\mathrm{Mi}$ (MOR) é de baixa potência, sendo seus efeitos analgésicos também associados ao aumento da disponibilidade de serotonina e noradrenalina nos pontos nervosos de controle da dor (SOUSA; ASHMAWI, 2015). 
Um possível problema encontrado no uso de opioides nessa unidade de saúde, é o grandenúmero de prescrição de morfina na concentração de $10 \mathrm{mg}$, pois o sulfato de morfina é um analgésico opioide forte, sistêmico, usado para o alívio da dor intensa, devendo ser reservado para as manifestações dolorosas mais graves, como no infarto do miocárdio, lesões graves ou dor crônica severa associada ao câncer terminal. Seu uso também é indicado na analgesia de pacientes sob ventilação mecânica, na suplementação da anestesia geral, regional ou local, na analgesia pósoperatória e para ma-nejo da dor aguda e em cuidados paliativos (RIPAMONTIet al., 2012).

\section{CONCLUSÃO}

Os estudos de utilização de medicamentos objetivam, segundo a Organização Mundialda Saúde (OMS), estudar a comercialização, distribuição, prescrição e uso de medicamentos na sociedade, com ênfase especial nas consequências médicas, sociais e econômicas (ANVISA, 2012).

Foi encontrado um grande uso de derivados opioides sendo prescritos em altas concentrações, mesmo para pacientes não internados, que receberiam um acompanhamento assistencial mais completo. É importante um programa de educação continuada para os profissionais de saúde para melhor selecionarem os medicamentos e saberem orientar sobre potenciais problemas relacionados a esta classe de substâncias.

Apesar do grande número dispensado dos produtos observados, não representaram gastos exorbitantes para sua obtenção, ficando o maior problema ligado a possíveis respostas negativas às terapias estabelecidas.

Os registros de dispensação seriam mais bem gerenciados com a utilização de um sistema informatizado que, além do quantitativo dos medicamentos direcionados para uso nas alas, fosse complementado com as informações de qual prescritor fez a solicitação, como também, as informações dos usuários. Tal medida 
daria um suporte mais adequado para a rastreabilidade geral da utilização dos medicamentos.

\section{REFERÊNCIAS BIBLIOGRÁFICAS}

AGÊNCIA NACIONAL DE VIGILÂNCIA SANITÁRIA. Boletim de Farmacoepidemiologia do SNGPC. Brasília: ANVISA, 2012.

AGÊNCIA NACIONAL DE VIGILÂNCIA SANITÁRIA. Câmara de Regulação do Mercado de Medicamentos - CMED. Brasília: ANVISA, 2020.

AGÊNCIA NACIONAL DE VIGILÂNCIA SANITÁRIA. Sistema Nacional de Gerenciamento de Produtos Controlados. Brasília: ANVISA, 2020.

BENSEÑOR, F. E. M.; CICARELLI, D. D. Sedação e Analgesia em Terapia Intensiva. Revista Brasileira de Anestesiologia. v. 53, n. 5, p. 680-93, 2003.

BICCA, C.; RAMOS, F. L.P.; CAMPOS, V. R.; ASSIS, F. D.; PULCHINELLI JR, A.; LERMNEN JR, N.; MARQUES, A. C. P. R.; RIBEIRO, M.; LARANJEIRA, R. R.; ANDRADA, N. C. Abuso e dependência dos opioides e opiáceos. Projeto Diretrizes. Associação Médica Brasileira, 2012.

BRASIL. Ministério da Saúde. Unidade de Pronto Atendimento (UPA). Brasília: MS, 2020. CASSETTARI, S. S. R.; MELLO, A. L. S. F. Demanda e tipo de atendimento realizado em Unidades de pronto atendimento do município de Florianópolis, Brasil. Texto Contexto Enfermagem. v.26, n. 1, sulpp. E3400015, 2017.

FEGADOLLI, C.; VARELA, N. M. D.; CARLINI, E. L. A. Uso e abuso de benzodiazepínicosna atenção primária à saúde: práticas profissionais no Brasil e em Cuba. Cad. Saúde Pública.v. 35, n. 6, sulpp. e00097718, 2019.

KRAYCHETE, D. C.; SIQUEIRA, J. D. D.; GARCIA, J. B. S. Recomendações para uso de opioides no Brasil: parte I. Rev. Dor. v.14, n. 4, p. 295-300, 2013.

MARTINS, R. T.; ALMEIDAI, D. B.; MONTEIROI, F. M. R.; KOWACSIV, P. A.; RAMINA, R. Receptores opioides até o contexto atual. Rev. Dor. v.13, n. 1, p. 75-9, 2012.

RIPAMOINTI, C. I.; SANTINI, D.; MARANZANO, E.; BERTI, M.; ROILA, F. Management of cancer pain: ESMO Clinical Practice Guidelines. Ann Oncol. v. 23, p. suppl 7:vii39-54, 2012.

SOUSA, A. M.; ASHAWI, H. A. O efeito analgésico de tramadol não é mediado por receptores opioides na dor de ratos no pós-operatório imediato. Rev Bras Anestesiol. v. 65, n. 3, p. 186190, 2015.

WOLRD HEALTH ORGANIZATION. Anatomical Therapeutic Chemical Code - ATC. Genebra: WHO, 2020.

ZWIELEWSKI, G.; BUB, T. F. Identificação de Transtornos de Ansiedade em Pacientes com Suspeita de Doença Arterial Coronariana. Rev Bras Cardiol. v. 27, n. 5, p. 311-313, 2014. 